\title{
Avances en educación superior: irrupción femenina y continuidad masculina
}

\author{
Silvia Luna Santos*
}

\begin{abstract}
Thanks to the expansion of the Mexican higher education system from the 1970s, the population with higher education studies has been increased, particularly the feminine one. This evidence has been obtained from a gender and generational analysis. The analysis also shows the socio-demographic characteristics of the population with higher education studies, which suggest that there are some advantages for women of the most recent generations, for instance not having become part of the labour market during their university studies. It is suggested that the fact that women have reached levels of higher education has generated a greater participation in the economic activity, which in turn allows them to have more autonomy and puts them in a better position to negotiate both in the family and labour spheres.
\end{abstract}

Keywords: higher education, gender, generations, institutions of higher education, university, undergraduate studies, postgraduate studies.

\section{Resumen}

Gracias a la expansión del sistema de educación superior mexicano, a partir de los años setenta, se ha incrementado la población con estudios superiores, particularmente la femenina. Esto se evidencia mediante un análisis por sexo y generaciones. Además, se muestran las características sociodemográficas de la población con educación superior, las cuales sugieren situaciones de ventaja para las mujeres de las generaciones más recientes como, por ejemplo, no haberse incorporado al mercado laboral durante el desarrollo de sus estudios universitarios. Se sostiene que el hecho de que las mujeres hayan alcanzado niveles de educación superior ha generado una mayor participación en la actividad económica lo que, en conjunto, les permite mayor autonomía y las pone en una mejor posición para negociar tanto en al ámbito familiar como en el laboral.

Palabras clave: educación superior, género, generaciones, instituciones de educación superior, universidad, licenciatura, posgrado.

* Dirección de Análisis y Estadística del Instituto Nacional de las Mujeres (Inmujeres). Correo-e: luna_silvia@hotmail.com. 


\section{Introducción $^{1}$}

La educación representa para las personas la posibilidad de adquirir conocimientos y desarrollar habilidades y capacidades, a fin de acceder al mercado de trabajo en actividades productivas y bien remuneradas, y de incorporarse a la vida social, económica y política del país. Por ello, entre otras cosas, la educación constituye el componente del desarrollo social que atenúa las disparidades sociales de manera más efectiva.

La tendencia de la dinámica poblacional anticipa, para los próximos años, una mayor demanda de educación media superior y superior por el esperado crecimiento de la población en edad laboral durante el primer decenio del siglo, sobre todo del grupo de 15 a 24 años, ${ }^{2}$ debido a la llegada de generaciones aún numerosas que nacieron en la todavía época de alta fecundidad. ${ }^{3}$ Esto advierte la necesidad de un aumento en la oferta educativa, particularmente la de nivel superior, dadas las expectativas crecientes de las mujeres y los hombres que hoy se encuentran incorporados en el sistema educativo.

En México, como en la mayoría de las sociedades industriales contemporáneas, es posible observar un aumento lento pero constante de las oportunidades educativas para sectores sociales antes excluidos. En efecto, la probabilidad de que mujeres y hombres jóvenes accedan actualmente a la educación y transiten exitosamente hasta el nivel universitario o superior ha aumentado, aun entre los sectores de escasos recursos económicos (Pérez Franco, 1998). Específicamente, la matrícula del sistema de educación superior creció a una tasa promedio anual de 4.5 en el periodo $1980-1990$ y de 5.7 entre 1990 y 2000. El periodo donde se observa el mayor crecimiento es 1994-2000, cuando la tasa promedio anual fue $7.4 .^{4}$

En la actualidad, la educación es valorada en todos los sectores de la sociedad y no sólo en las capas medias y altas. Esto se debe, por un lado, a la movilidad social que han mostrado las

\footnotetext{
${ }^{1}$ Agradezco a Israel Laguna por el procesamiento de la información y la presentación gráfica.

${ }^{2}$ Este grupo poblacional se calculaba, en el año 2000, en 20.3 millones de personas, y se espera que en el 2010 alcance su máximo histórico, previsto en 21.2 millones (SEP, 2001).

${ }^{3}$ La tasa global de fecundidad a mediados de la década de 1970 era de alrededor de seis hijos por mujer; para 1985 se había reducido a cuatro hijos (Tuirán, 2002).

${ }^{4}$ Los cálculos de estas tasas promedio anual de crecimiento fueron realizados con base en los Anuarios Estadísticos de ANUIEs, 1980-2000.
} 
personas con altos niveles de instrucción y a las mayores dificultades para obtener empleos relativamente bien remunerados para quienes no tienen las calificaciones necesarias o los certificados formales y, por el otro lado, a la ampliación de la infraestructura y oportunidades educativas. No obstante, persisten obstáculos que impiden acceder a la educación superior, permanecer en ella y graduarse oportunamente. De acuerdo con datos de la SEP (2001), $45 \%$ de las personas de 19 a 23 años que vive en zonas urbanas y pertenece a familias con ingresos medios o altos recibe educación superior, mientras que sólo $11 \%$ de quienes habitan en sectores urbanos pobres y $3 \%$ de los que viven en sectores rurales pobres tiene acceso a este tipo de estudios.

El proceso de expansión de las oportunidades educativas, particularmente en el nivel superior, comenzó en los años setenta, tuvo un momento de aceleración que coincidió con el auge del desarrollo y que continuó incluso hasta el inicio de la crisis de los ochenta. Esto fue resultado de la política educativa de los setenta, la cual planteaba que la educación era una condición necesaria para el desarrollo y que el Estado tenía la obligación de abrir oportunidades para todos en ese sentido. Además, la creciente valoración de la educación por parte de la población se tradujo en una mayor demanda de servicios educativos, que incluía los de nivel superior.

La mayor demanda de servicios educativos también tuvo que ver con factores sociodemográficos, dado que el volumen de población que en los setenta tenía la edad y la intención de ingresar a las instituciones de educación superior reflejaba, por un lado, la elevada fecundidad de los años cincuenta y sesenta y, por otro, una proporción importante de población que para la década de los setenta había terminado la educación media superior, particularmente en las ciudades.

Para muchos individuos resulta indispensable tener la posibilidad de combinar el trabajo con los estudios. Sin embargo, la realidad ha mostrado que combinar estas actividades no es fácil, particularmente en situaciones de crisis económicas, puesto que cambian los costos de oportunidad y las opciones tienden a ser excluyentes. Esto se evidencia en los altos índices de deserción escolar y la baja eficiencia terminal. En este sentido, la SEP (2001) reconoce que sólo 50\% de los estudiantes de licenciatura y alrededor de $40 \%$ de los de posgrado concluyen sus estudios y se titulan, lo cual representa un desperdicio de recursos y la frustración de aspiraciones. 
Para analizar las situaciones que compiten con el ingreso, permanencia y conclusión exitosa de la educación superior, varios estudios se han dedicado a estudiar los aspectos familiares de tipo socioeconómico y cultural de la población estudiantil, ya que ellos constituyen el escenario en el que se desenvuelven y el entorno que les permite llevar a cabo y concluir su formación educativa. El primer tipo incluye características como el ingreso del hogar, el número de personas dependientes de ese ingreso, las condiciones de la vivienda que se habita, la composición familiar y las categorías ocupacionales de los padres, mientras que el aspecto cultural de las familias se identifica a través del nivel de escolaridad de los padres, la valoración de la educación y el acceso a bienes culturales como libros, revistas y juegos, entre otros.

Otros estudios incluyen condicionantes del contexto socioeconómico. Con ellas, se busca reflejar las transformaciones socioeconómicas que afectan tanto a las instituciones como a las familias y los individuos. En su estudio sobre dos cohortes de ingreso a la Universidad Autónoma Metropolitana (1979 y 1987), Muñiz (1997) utiliza como indicador resumen del contexto socioeconómico el año de ingreso a la universidad, con el que pretende señalar la realidad macrosocial del país en esos años.

En general, todos los estudios incluyen las características sociodemográficas de la población que está formándose en el nivel superior, tales como el sexo, la edad al ingresar a la universidad, el estado civil, la dependencia o participación económica, el tiempo dedicado al trabajo, ya que son factores determinantes para el acceso y desarrollo de los estudios de nivel superior. Por ejemplo, dan cuenta de eventos que compiten tanto con el ingreso como con la permanencia de la población en ese nivel de formación escolar.

Este artículo busca analizar, por un lado, los cambios en la participación de mujeres y hombres en la formación de nivel superior, a lo largo de las últimas décadas y, por el otro, caracterizar a la población que cuenta con al menos un año cursado de educación superior, en términos sociodemográficos.

Los estudios revisados sobre población en el nivel superior de instrucción se dedican, sobre todo, al análisis de los estudiantes inscritos en las instituciones de educación superior a partir de los registros que sobre ellos cuentan las propias instituciones. Este texto pretende ser más amplio, aunque menos específico y, por ello, incluye a toda la población que en el 2000 declaró que contaba con al menos un año concluido de educación superior. Esto permitirá evaluar los cambios en el tiempo respecto al com- 
portamiento y el perfil de las mujeres y los hombres que han logrado acceder a ese nivel de instrucción.

Además, a través de un análisis por generación y con el supuesto de una edad 'estándar' de ingreso a la universidad (alrededor de 20 años), ${ }^{5}$ podremos identificar el momento en que se realizaron los estudios y, por tanto, las condiciones macrosociales que pudieron haber incidido en el acceso a la formación superior de mujeres y hombres.

\section{1. ¿Hay un solo momento para formarse en el nivel superior?}

El crecimiento de la matrícula a partir de la década de los setenta, particularmente en los primeros niveles de instrucción, y la consecuente complejidad administrativa que generó para la administración del sistema educativo, presionó para que se normara la trayectoria escolar. Entre las medidas más importantes que se tomaron en este sentido se encuentra la regularización de la edad de ingreso a educación primaria, la cual ha tendido a homogeneizar las edades en las distintas etapas educativas hasta la salida de la educación preuniversitaria (Muñiz, 1997).

Como resultado de lo anterior, en general se considera a la población de edades entre los 18 y los 24 años como la adecuada para medir las tasas de participación educativa en el nivel superior que incluye profesional o licenciatura y posgrado (maestría y doctorado). Sin embargo, esto no resulta del todo pertinente, ya que, de acuerdo con la información del XII Censo General de Población y Vivienda, 2000, sólo 52\% de los hombres y 61\% de las mujeres pertenecen a ese grupo de edad entre quienes asisten a la universidad en el nivel de licenciatura o profesional (véase la gráfica I). Por ello, creemos que un análisis exhaustivo de la población que está formándose profesionalmente debe incluir a todos los implicados, aun cuando rebasen los 24 años. $^{6}$

En México, 43.5\% de la población estudiante en ese nivel de instrucción es mayor de 24 años y, por diversas situaciones, ha decidido o encontrado la posibilidad de entrar a la universidad más tarde de lo usual o lo esperado. Incluso, es posible que el

\footnotetext{
${ }^{5}$ Recientemente, la edad de ingreso a la universidad parece ubicarse entre los $20 \mathrm{y}$ 21 años, de acuerdo con el estudio que Muñiz (1997) elaboró para dos cohortes de la Universidad Autónoma Metropolitana.

${ }^{6}$ De hecho, la tasa bruta de matriculación terciaria, propuesta por la UNESCO, se calcula dividiendo el número de estudiantes matriculados en un nivel de enseñanza, sea cual fuere su edad, entre la población en edad escolar oficial para ese nivel (PNUD, 2001).
} 


\section{Gráfica I}

Distribución porcentual de la población de 18 años y más con asistencia escolar a la educación superior* según grupos de edad, por sexo, 2000

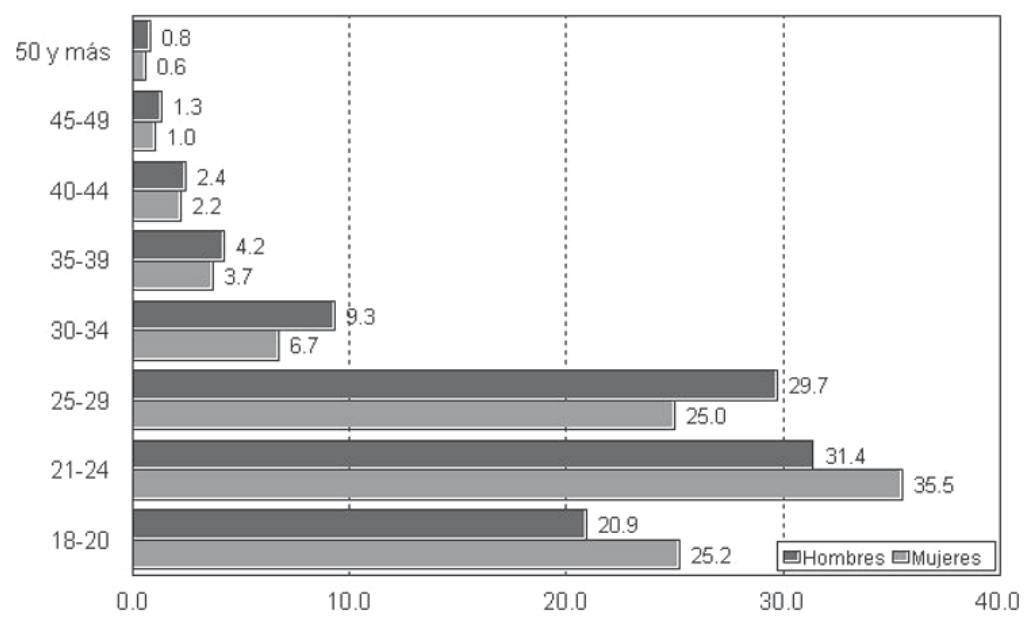

* Incluye carrera técnica o comercial con antecedentes de preparatoria terminada. Fuente: Reprocesamiento con base en la muestra censal del XII Censo General de Población y Vivienda, 2000.

ingreso de este sector haya ocurrido una vez que ha experimentado otros eventos tales como la unión y la paternidad. Particularmente, destaca el grupo de 25 a 29 años de edad que casi representa $30 \%$ de la población adscrita a la universidad. Creemos que esa elevada proporción puede estar dando cuenta de suspensiones temporales durante el bachillerato o la propia licenciatura, debidas a inserciones en el mercado de trabajo.

Reconocemos que una vez que los universitarios(as) suspenden los estudios debido a su inserción en el mercado de trabajo, su regreso a la universidad se vuelve difícil si ello implica dejar de obtener ingresos. No obstante, es posible que algunos de ellos(as) sean motivados por las presiones del mercado laboral por mayores cualificaciones, lo cual se evidencia en el cada vez más temprano ingreso a estudios de posgrado. En ese sentido, cobra importancia la diversificación de las opciones para la titulación que ha venido observándose en los últimos años, así como los sistemas de educación abierta y la educación a distancia. 


\section{Población con educación superior profesional y de posgrado}

En los estudios de cohortes universitarias, el año de ingreso a la educación superior se utiliza como el indicador que permite dar cuenta del contexto social que favorece o no la entrada y permanencia en la universidad. Dado que nuestro estudio no utiliza datos de la matrícula universitaria sino la población que en el censo del 2000 declaró haber cursado al menos un año de formación superior, sólo utilizando una edad promedio de entrada a la universidad (alrededor de los 20 años), podemos aproximarnos a las condicionantes socioeconómicas que hubieran podido incidir en la posibilidad de ingresar y permanecer en las instituciones de educación superior.

Además, al hacer un análisis por generaciones, buscamos poner en relieve aquellos grupos de población que tuvieron más o menos posibilidades de acceder a la formación universitaria, aunque esto no haya sido dentro de una trayectoria regular, es decir, que su ingreso no haya sido inmediatamente después de la salida de la preparatoria o vocacional (entre los 18 y 20 años). De ese modo, es posible relacionar, en buena medida, la magnitud del acceso a la universidad con las distintas oportunidades económicas y sociales que han caracterizado la vida en México en las últimas décadas.

\subsection{Educación superior profesional}

Una forma de aproximarnos a la población que tuvo la oportunidad de cursar al menos un año de educación superior es a través de lo que hemos denominado tasas de acceso a educación superior, las cuales relacionan a la población con al menos un año completo de educación superior -en el nivel profesional o de licenciatura- con la población total 'en riesgo de recibirla'. Al observar el comportamiento de estas tasas por grupos generacionales, destaca un creciente acceso a la enseñanza universitaria, particularmente entre las mujeres, lo cual coincide con hallazgos previos. Para el periodo entre 1989 y 1997, se calculó que la presencia femenina en el nivel licenciatura creció a una tasa media anual de $6.0 \%$, mientras que la presencia masculina apenas lo hizo a un ritmo de $2.3 \%$ anual en el mismo periodo (Conmujer et al., 2001).

Las tasas de acceso a la educación superior profesional más elevadas corresponden a los hombres nacidos entre 1955 y 1969 , 


\section{Gráfica II \\ Tasa de acceso a la educación superior profesional* por generación y sexo, 2000}

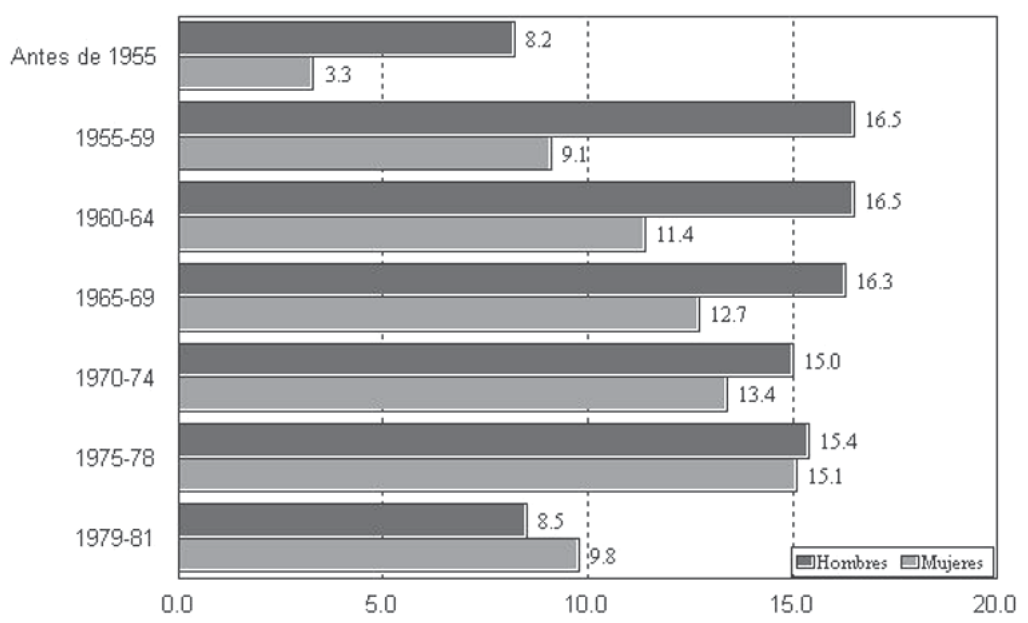

* Incluye carrera técnica o comercial con antecedentes de preparatoria terminada. Fuente: Reprocesamiento con base en la muestra censal del XII Censo General de Población y Vivienda, 2000.

mientras que en la generación más joven (1979-1981), las tasas femeninas de acceso a la educación superior profesional rebasan las masculinas. Esto parece obedecer a que las mujeres siguen, recientemente y con mayor frecuencia, trayectorias regulares en términos de desarrollo escolar y, por ello, su participación es más amplia en edades tempranas de ingreso a la educación superior.

Al realizar el análisis de las tasas de acceso a la educación superior profesional por último año cursado entre aquellos con asistencia escolar en el 2000, encontramos mayores tasas femeninas en el primer año de educación superior profesional en la población cuya edad oscilaba entre 18 y 20 años en el $2000^{7}(5.1$ y $4.6 \%$, respectivamente, para mujeres y hombres). En el segundo año, la tasa femenina sigue siendo mayor para el mismo grupo de edad (2.9 y $2.4 \%$, respectivamente, para mujeres y hombres), no así para la población que en el 2000 tenía de 21 a 24 años: ${ }^{8}$ la tasa masculina de acceso es de $2.6 \%$ frente a la femenina de $2.0 \%$.

El conjunto de los datos analizados sugiere que las mujeres ingresan a la educación superior profesional más jóvenes que los

7 Generaciones 1979-1981.

${ }^{8}$ Generaciones 1975-1978. 


\section{Gráfica III}

Tasa de acceso a la educación superior profesional* de la población de 18 a 24 años por último año cursado de la población con asistencia escolar en el 2000, grupos de edad y sexo

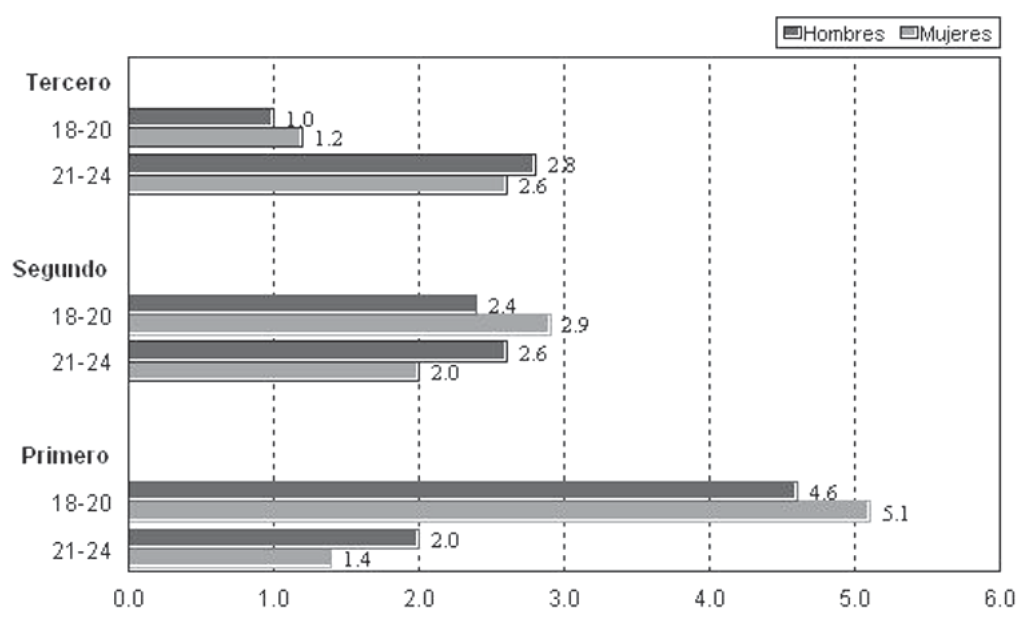

* Incluye carrera técnica o comercial con antecedentes de preparatoria terminada. Fuente: Reprocesamiento con base en la muestra censal del XII Censo General de Población y Vivienda, 2000.

hombres. Esto coincide con hallazgos de otras investigaciones en el sentido de que las mujeres presentan, en comparación con los varones, una mayor continuidad en sus trayectorias estudiantiles. De hecho, se ha observado que las mujeres, en su mayor proporción, ingresan a la universidad entre los 18 y 19 años, lo que, según Muñiz (1997), puede ser el resultado de la institucionalización o normatividad, cada vez más marcada, en cuanto a la edad al ingreso a la vida escolar. Esto se traduce en trayectorias previas 'regulares'; no obstante, llama la atención el hecho de que sean las mujeres quienes se ajustan más a ese patrón 'normalizado'.

Las tasas de acceso al tercero y cuarto año de educación superior profesional entre aquellos con asistencia escolar en el 2000 y correspondientes a la población de 21 a 24 años ${ }^{9}$ son ligeramente superiores para la población masculina en relación con la femenina: 2.8 y $2.6 \%$ en el tercer año, respectivamente, para hombres y mujeres, y 3.1 y $3.0 \%$ en el cuarto año. 
$\mathrm{Al}$ analizar el cuarto año cursado de estudios universitarios, conviene empezar a poner atención en la población que ya no asiste a formarse a las instituciones de educación superior, la cual debe corresponder a aquella egresada de las distintas carreras universitarias, se haya o no titulado. Las mayores tasas de acceso al cuarto año de educación superior profesional corresponden a la población masculina de las generaciones de 1955 a 1964 (alrededor de seis de cada 100). Las mujeres alcanzan el nivel de las tasas masculinas sólo a partir de la generación 1965 e incluso las rebasan a partir de la generación 1970.

\section{Gráfica IV}

Tasa de acceso al cuarto año de educación superior profesional* de la población que no asiste a la escuela en el 2000 , por generación y sexo

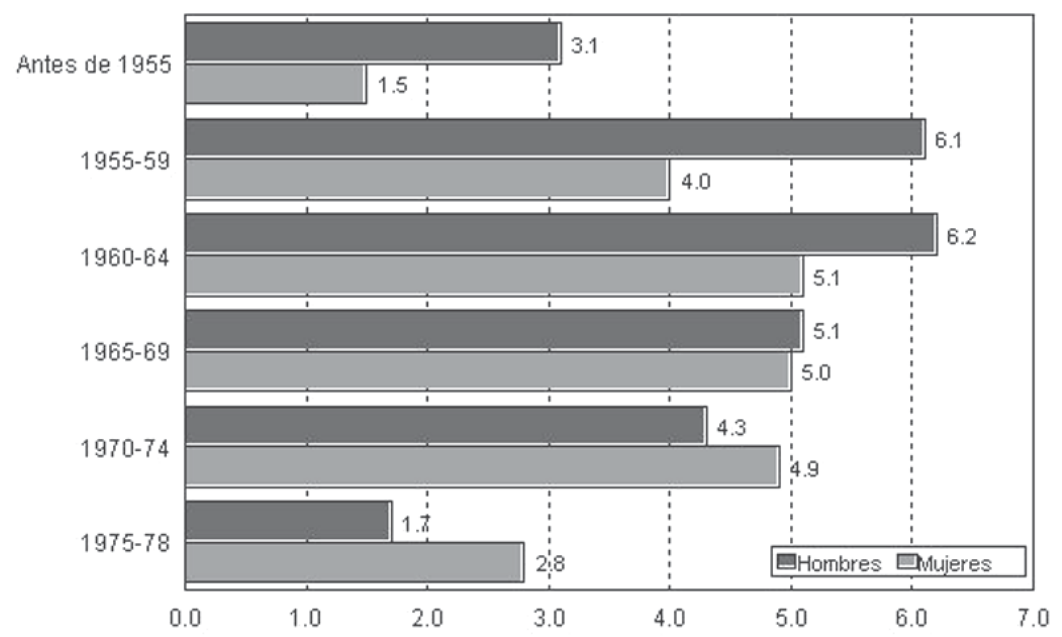

* Incluye carrera técnica o comercial con antecedentes de preparatoria terminada. Fuente: Reprocesamiento con base en la muestra censal del XII Censo General de Población y Vivienda, 2000.

Si el hecho de haber detenido la educación superior en el cuarto año significa la conclusión, el mejor desempeño observado de las mujeres entre las generaciones más jóvenes puede explicarse, como se verá más adelante, por la tendencia femenina a no abandonar los estudios sin concluirlos y a la mayor frecuencia con que las mujeres se titulan respecto de los hombres. ${ }^{10}$

${ }^{10}$ En el año 2000, el número de mujeres egresadas de licenciatura fue mayor que el de hombres: 104,996 y 104,799, respectivamente. El mismo comportamiento se observa entre los titulados: 68,745 mujeres y 66,488 hombres (ANUIES, 2002). 
Cuando se trata de la población que alcanzó un quinto o sexto grado de educación profesional, y que ya no asiste a las instituciones de educación superior, las tasas son más altas entre la población masculina en todas las generaciones anteriores a 1975. Las tasas femeninas se acercan mucho a las masculinas en las generaciones de 1970 a 1974, y las correspondientes a las generaciones 1975 a 1978 llegan a rebasarlas (véase la gráfica v). Esto muestra que además de una mayor participación de la mujer en la formación superior profesional, ésta se refiere no sólo al acceso sino también a la permanencia en carreras de larga duración.

\section{Gráfica $\mathrm{V}$}

Tasa de acceso al quinto o sexto año de educación superior profesional* de la población que no asiste a la escuela en el 2000 , por generación y sexo

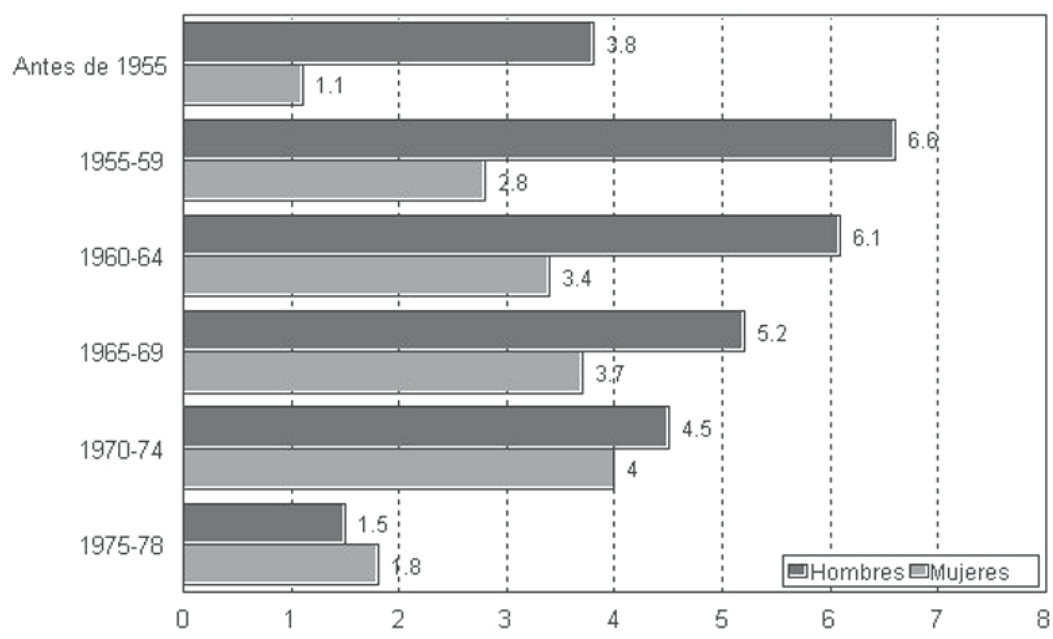

* Incluye carrera técnica o comercial con antecedentes de preparatoria terminada. Fuente: Reprocesamiento con base en la muestra censal del XII Censo General de Población y Vivienda, 2000.

\subsection{Posgrado}

Cuando se trata del nivel de maestría o doctorado, las tasas de acceso a ese nivel de educación son sensiblemente menores a las de licenciatura o profesional y, entre ellas, las más altas se observan en las generaciones de 1950-1964. En parte, esto puede explicarse porque, en el pasado, la realización de ese tipo de estudios 
suponía, además de una formación universitaria previa, una probada experiencia profesional, lo que implicaba iniciar estudios de maestría en edades avanzadas. Sin embargo, es posible que en el corto plazo notemos cambios en este comportamiento y encontremos población más joven con niveles de posgrado debido a la mayor continuidad en los estudios superiores presentada entre las últimas generaciones con educación superior. Actualmente se estudia una maestría inmediatamente después de concluir la licenciatura y, a veces, esa secuencia llega hasta el doctorado. Esta nueva tendencia puede ser una respuesta a la escasa oferta de empleos bien remunerados, ${ }^{11}$ así como a la presión del mercado laboral que demanda mayores cualificaciones, es decir, a la expectativa de que una mayor formación profesional permitirá obtener un mejor trabajo.

Los niveles observados de las tasas de acceso a estudios de posgrado se explican, mayoritariamente, por la población masculina que ha cursado algún año de ese nivel de estudios $(1.4 \%$ de hombres en las generaciones de 1950 a 1959, y 1.0\% en las de 1960 a 1964). Sin embargo, llama la atención el hecho de que la distancia de las tasas de las generaciones femeninas de 1965 a 1977 se va acortando en relación con las masculinas, lo que sugiere que las mujeres empiezan a formarse, con mayor frecuencia, en esos niveles de estudio, seguramente siguiendo la tendencia reciente de realizar estudios de posgrado inmediatamente después de los de licenciatura.

\section{El acceso a la educación superior en el contexto internacional}

A pesar de los indicios de un mayor ingreso a la educación superior entre la población joven, tanto entre hombres como mujeres, es necesario evaluar qué representa ese avance para la población mexicana y dónde sitúa al país en términos de formación de capital humano. Para ello presentamos, para países seleccionados, el porcentaje de la población de 25 a 34 años que ha alcanzado la educación superior o terciaria ${ }^{12}$ respecto de la po-blación total de 25 a 34 años, la cual, de haber seguido una trayectoria

\footnotetext{
${ }^{11}$ De acuerdo con la Encuesta Nacional de Empleo de 2000, 3 de cada 4 personas de la población ocupada reciben un ingreso menor a los 5 salarios mínimos y, peor aún, 6 de cada 10 personas ocupadas reciben un ingreso menor a los 3 salarios mínimos.

${ }^{12} \mathrm{La}$ enseñanza terciaria es la educación que se ofrece en universidades y escuelas normales superiores de formación profesional de nivel superior.
} 


\section{Gráfica VI \\ Tasa de acceso a estudios de posgrado por generación y sexo, 2000}

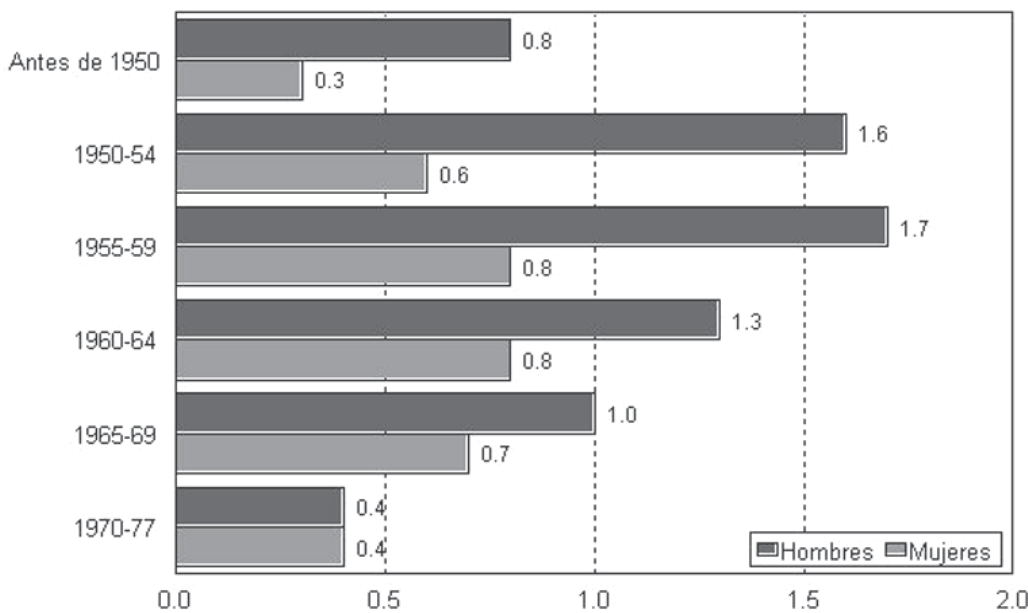

Fuente: Reprocesamiento con base en la muestra censal del XII Censo General de Población y Vivienda, 2000.

regular de estudios, debió haber concluido su formación profesional en las últimas dos décadas.

Dentro del marco internacional, este porcentaje es bajo en nuestro país: en 2001, 20\% de los hombres y 16\% de las mujeres, ambos de 25 a 34 años, habían obtenido instrucción superior, mientras que en Canadá, país calificado con un alto desarrollo humano, esos porcentajes fueron 45 y 56, para mujeres y hombres, respectivamente (véase el cuadro 1). Hay que reconocer, no obstante, que los países industrializados cuentan con una ventaja histórica acumulada en materia de formación de capital humano, mientras que en México esa experiencia se remonta sólo a unas cuantas décadas, particularmente a partir de la fundación del Instituto Politécnico Nacional, la de Ciudad Universitaria y las universidades de provincia, las universidades autónomas metropolitanas y el sistema privado de educación superior.

La comparación de México respecto de Canadá, país con el mayor porcentaje de población con educación terciaria, no sólo destaca la diferencia en la magnitud (25 y 40 puntos porcentuales respectivamente para hombres y mujeres), sino también la ventaja femenina. Esto puede ser relevante, ya que en todos los países donde se ha incrementado el porcentaje de población de 25 


\section{Cuadro 1}

Porcentaje de la población de 25 a 34 años que alcanzó la educación terciaria* en países seleccionados, por sexo, 1991-2001

\begin{tabular}{lccc}
\hline \multicolumn{2}{c}{ País /año } & Hombres & Mujeres \\
\hline Canadá & 1991 & 30 & 33 \\
& 2001 & 45 & 56 \\
Estados Unidos & 1991 & 29 & 31 \\
& 2001 & 36 & 42 \\
España & 1991 & 15 & 18 \\
& 2001 & 32 & 39 \\
Francia & 1991 & 19 & 21 \\
& 2001 & 32 & 37 \\
México & 1991 & nd & nd \\
& 2001 & 20 & 16 \\
Reino Unido & 1991 & 19 & 29 \\
\hline
\end{tabular}

* No se especifica si se trata de egresados, titulados o simplemente de aquellos que terminaron al menos un año de educación superior.

Fuente: www.oecd.org/dataoecd/1/38/14158913.xls, 24 de agosto de 2004.

a 34 años con educación terciaria, en la última década, las mujeres han aventajado a los hombres. La excepción es el Reino Unido, donde todavía la población masculina de 25 a 34 años ha mantenido mayores niveles de educación superior respecto de las mujeres, aunque con una diferencia de apenas un punto porcentual. Actualmente, México se encuentra en los niveles que España presentaba en 1991. En 10 años, ċalcanzaremos los niveles de España en 2001? ¿Cuál es el camino que México seguirá respecto de la formación de su población en el nivel de educación superior?

Para responder las preguntas planteadas, no sólo hay que considerar que en México menos de una quinta parte de la población joven, de 25 a 34 años, accede a la educación superior, sino también al hecho de que una proporción importante de ella no la concluye.

\section{Una aproximación a la deserción}

La deserción escolar, así como la reprobación y el consecuente rezago que caracteriza a un porcentaje no despreciable de las 
trayectorias estudiantiles, en todos los niveles de instrucción, constituyen problemas importantes en nuestro país. Aun cuando la eficiencia terminal ha mejorado en los últimos años, la SEP (2001) reconoce que sólo $50 \%$ de los estudiantes de licenciatura y alrededor de $40 \%$ de los de posgrado concluyen sus estudios y se titulan, lo cual representa un desperdicio de recursos y la frustración de aspiraciones.

De acuerdo con hallazgos de otros estudios, el número de personas que no llega a concluir su formación profesional superior ha aumentado en forma considerable. En las cohortes de estudiantes de 1979 y 1987 de la Universidad Autónoma Metropolitana, casi la mitad son desertores (Muñiz, 1997). Sin embargo, esta situación no sucede igualmente entre mujeres y hombres. En efecto, algunos estudios han dado cuenta de esta situación como el desarrollado por Díaz et al. (2000) sobre eficiencia terminal de las instituciones mexicanas de educación superior en 1999. En él encuentran que las mujeres terminan sus estudios en $49 \%$ de los casos frente a $45 \%$ observado entre los hombres. Este porcentaje se incrementa en el caso de las universidades de corte tecnológico, tanto para la población femenina como la masculina; no obstante, en 1999 las mujeres siguieron presentando una mayor eficiencia terminal $(56 \%$ y $50 \%$, respectivamente, para mujeres y hombres). El análisis que se presenta a continuación confirma estos hallazgos.

Considerar en conjunto a las personas que ya no asisten a formarse a las instituciones de educación superior, pero que al menos han cursado un año de estudios superiores profesionales, implica mezclar personas que concluyen su formación profesional satisfactoriamente y que se titulan con aquellas que sólo egresan de las distintas carreras y aquellas que abandonan los estudios. A fin de distinguir estas dos poblaciones y, sobre todo, en el afán de conocer la magnitud del fenómeno de la deserción de los estudios superiores, nos aproximamos a este último tema cuando el abandono de los estudios profesionales sucede antes de la mitad de la duración media de una carrera universitaria, esto es, antes de haber cursado el tercer año en la universidad.

Para la población sin asistencia escolar en el 2000 y con al menos un año de estudios profesionales, 4.2 de cada mil hombres y 2.7 de cada mil mujeres cuentan con un solo año cursado. Al analizar esta situación por generación, se observa que la mayor deserción de la población con sólo un grado de estudios es mayor en las 


\section{Cuadro 2 \\ Tasa de acceso a la educación superior profesional* por último año cursado de la población que no asiste a la escuela en 2000, generación y sexo}

\begin{tabular}{rrrr}
\hline \multicolumn{2}{c}{ Año / generación } & Mujeres & Hombres \\
\hline Primero & & & \\
& Antes de 1955 & 0.6 & 1.7 \\
& $1955-59$ & 2.8 & 5.4 \\
$1960-64$ & 3.5 & 6.1 \\
$1965-69$ & 4.4 & 8.3 \\
$1970-74$ & 4.0 & 5.1 \\
$1975-78$ & 3.8 & 4.1 \\
& $1979-81$ & 2.7 & 2.0 \\
Segundo & & & \\
& Antes de 1955 & 1.2 & 3.2 \\
& $1955-59$ & 4.2 & 9.9 \\
& $1960-64$ & 5.4 & 10.1 \\
$1965-69$ & 7.5 & 7.1 \\
$1970-74$ & 5.6 & 4.7 \\
& $1975-78$ & 4.6 & 1.6 \\
\hline
\end{tabular}

\footnotetext{
* Incluye carrera técnica o comercial con antecedente de preparatoria terminada. Fuente: Reprocesamiento con base en la muestra censal del XII Censo General de Población y Vivienda, 2000.
}

generaciones de 1965 a 1969. Este comportamiento es mucho más acentuado entre los hombres (véase el cuadro 2).

En el segundo año, la deserción es aún más importante, particularmente en las generaciones de 1955 a 1969. Nuevamente, son los hombres quienes muestran una deserción mayor: duplican la de las mujeres en las generaciones anteriores a la de 1970.

Conviene recordar aquí que al analizar la evolución de las tasas de acceso a la educación superior profesional encontramos incrementos importantes hasta la generación de 1969, y para las generaciones posteriores percibimos un ligero descenso, el cual puede estar asociado con los efectos de la crisis de 1982, esto es, la disminución del ingreso de los hogares. Como han demostrado Cortés y Ruvalcaba (1991), la crisis de los años ochenta del siglo xx indujo a que un número mayor de miembros del hogar se integrara a la actividad económica, lo cual seguramente implicó sacrificar la oportunidad de que la población en edad de formarse en un nivel profesional ingresara o permaneciera en las instituciones de educación superior. Esto mismo podría explicar también la más alta deserción después de uno o dos años cursados 
de educación superior en las generaciones de 1965 a 1969, que en una trayectoria regular hubieran realizado sus estudios profesionales entre 1985 y 1989.

La información aquí analizada nos lleva a reflexionar sobre dos situaciones. La primera tiene que ver con el hecho de que las mujeres abandonan con menor frecuencia su formación profesional. En efecto, se ha encontrado que la población femenina presenta una menor tendencia a la deserción y una mayor tendencia a titularse (Díaz et al., 2000 y Muñiz, 1997). En su estudio sobre dos cohortes de estudiantes de la Universidad Autónoma Metropolitana, Muñiz (1997) señala que el porcentaje de mujeres que se titula duplica al de los hombres y que la proporción de ellas entre los desertores es menor. La misma autora sugiere que el mejor desempeño de las mujeres puede explicarse porque, en una situación de crisis económica, los roles tradicionales de género llegan a favorecerlas, ya que se espera que sean los hombres quienes primero participen en la actividad económica para contribuir al ingreso del hogar. Ello resulta, de manera indirecta, en una mayor permanencia de las mujeres en la educación superior, muchas veces de tiempo completo. Las diferencias por sexo en la participación de la actividad económica encontradas entre la población que en el 2000 estudiaba el nivel profesional a la vez que trabajaba (55.0 y 42.9\% de hombres y mujeres, respectivamente) dan cuenta de lo anterior.

Por tanto, aun cuando la educación de los hombres es vista como un requerimiento indispensable para cumplir mejor su rol de proveedores principales del hogar, las crisis económicas de las últimas décadas los han obligado a desertar de la escuela e ingresar anticipadamente en el mercado de trabajo para que empiecen a cooperar en el sostenimiento económico familiar. Por el contrario, debido al menor valor social otorgado a la educación superior de las mujeres, la mayoría de quienes logran acceder a ese nivel ha sido después de haber pasado un filtro social importante, por lo que su permanencia en la escuela presenta menores riesgos (Conmujer et al., 2001).

La segunda situación se refiere a la ventaja que da a la población haber cursado uno o dos años de educación superior profesional. Para muchos, adquirir una capacidad profesional básica uno o dos años de alguna carrera universitaria, por ejemplo- puede implicar el contacto con el medio profesional de su área de estudio. Así, contar con uno o dos años de educación superior les permite integrarse a una red profesional en la que pueden encontrar 
un trabajo que, eventualmente, les dará experiencia. Por tanto, aun cuando los 'desertores' no vuelvan a las instituciones de educación superior a obtener el grado, contar con una formación profesional mínima les permite acceder a mejores empleos.

\section{5. Áreas de estudio}

Si consideramos que la población con al menos un año de educación superior sin asistencia escolar en el 2000 -ya sea por conclusión o abandono de los estudios- corresponde a las generaciones más viejas, y que la población que en el 2000 asistía a las instituciones de educación superior pertenece a las generaciones más jóvenes, encontramos diferencias importantes en lo que estudiaba una y otra población, al hacer el análisis por sexo.

El índice de feminidad ${ }^{13}$ entre la población que en el 2000 asistía a las instituciones de educación superior, por áreas de estudio, es más alto en todos los casos respecto del observado para aquella población que ya no asistía en ese año (véase la gráfica VII). Esto destaca particularmente en ciencias humanísticas (E), químicas (F), economía-administración-contaduría-turismo (I), ciencias de salud, nutrición y biomédicas (D), disciplinas artísticas $(\mathrm{H})$ y arquitectura-urbanismo-diseño industrial, de interiores, textil y gráfico $(\mathrm{A})$.

Entre la población que asistía en el 2000 a las instituciones de educación superior, encontramos que por cada 100 hombres inscritos en el área de educación y pedagogía, había 112 mujeres. Esta relación es mayor en el área de ciencias de la salud, nutrición y biomédicas y en ciencias humanísticas (113.5 y 128.6, respectivamente). En el resto de las áreas, las mujeres son menos que los hombres.

En general, se observa un incremento sustancial en la participación femenina en todas las áreas de estudio, aun en matemáticas, física y astronomía (L), ingenierías $(\mathrm{K})$ y ciencias agropecuarias, forestales y pesqueras $(\mathrm{C})$, donde los aumentos fueron muy pequeños. Estas últimas áreas han sido, tradicionalmente, las menos feminizadas, lo cual requiere una profunda investigación para conocer las razones por las cuales las mujeres deciden no incursionar en ellas, sobre todo en un contexto de mayor participación femenina en la formación profesional y dado que ellas

\footnotetext{
${ }^{13}$ El índice de feminidad resulta de dividir la población femenina entre la población masculina. En este caso, se calcula para la población con al menos un año de educación superior por área de estudio y lo expresamos por 100.
} 


\section{Gráfica VII}

Índice de feminidad de la población de 18 años y más con educación superior* por áreas de estudio y condición de asistencia, 2000

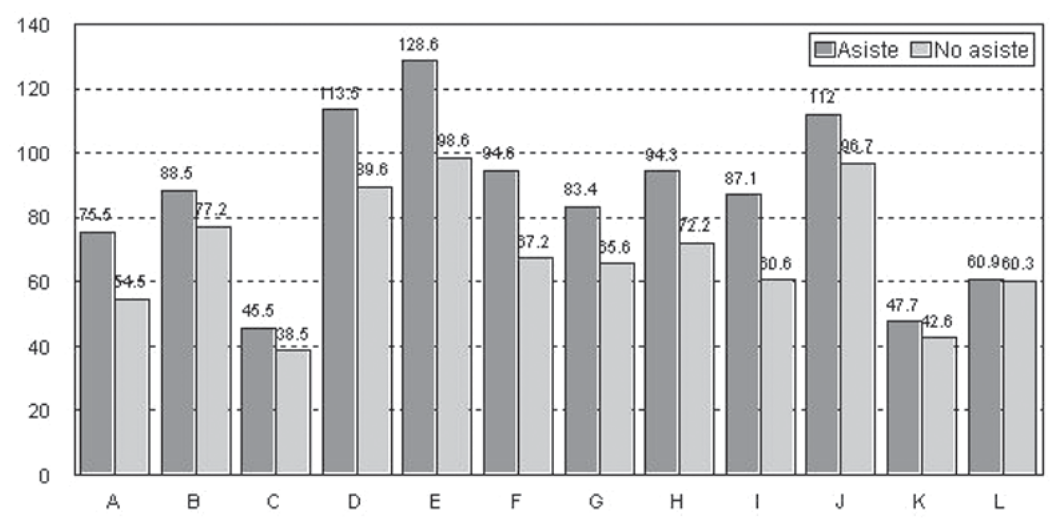

* Incluye carrera técnica o comercial con antecedentes de preparatoria terminada. Fuente: Reprocesamiento con base en la muestra censal del XII Censo General de Población y Vivienda, 2000.

A) Arquitectura, urbanismo, diseño industrial, de interiores, textil y grafico.

B) Biología, biotecnología, ecología, ingeniería ambiental, ciencias atmosféricas y ciencias del mar.

C) Ciencias agropecuarias, forestales y pesqueras.

D) Ciencias de la salud, nutrición y biomédicas.

E) Ciencias humanísticas.

F) Ciencias químicas.

G) Ciencias sociales, políticas, administración pública, relaciones internacionales, comunicación, derecho y geografía.

H) Disciplinas artísticas.

I) Economía, administración, contaduría y turismo.

J) Educación y pedagogía.

K) Ingenierías (civil, extractiva, metalúrgica, computación, informática, eléctrica, electrónica, mecánica, industrial, transportes, aeronáutica y topográfica).

L) Matemáticas, física y astronomía.

presentan, muchas veces, mejores rendimientos que los hombres (mayor titulación, por ejemplo).

\section{Situación familiar de la población con educación superior}

La situación familiar de la población que asiste a las universidades es fundamental, ya que enmarca los esfuerzos de los individuos y el ambiente que les permite o no llevar a cabo su formación educativa y profesional. En este sentido, nos interesa destacar, por un lado, el estado conyugal de quienes asistían en 
el 2000 a la universidad y, por el otro lado, su parentesco con el jefe de hogar. Una situación favorable para quienes asisten a la universidad sería aquella en que se es soltero e hijo, ya que ello puede implicar una dependencia económica respecto de los padres y, por tanto, la posibilidad de dedicarse por completo a los estudios superiores. ${ }^{14}$

\subsection{Estado conyugal}

Entre la población con asistencia escolar en el 2000 en el nivel profesional, cuatro de cada cinco eran solteros(as), mientras que la mayoría de aquellos que tienen un nivel de maestría y que en el 2000 asistían a las instituciones de educación superior, son casados cuando se trata de los hombres, mientras que entre las mujeres la proporción de solteras es prácticamente la misma que la de unidas (véase la gráfica VIII).

\section{Gráfica VIII}

Distribución porcentual de la población de 18 años y más con asistencia escolar en el 2000 según estado conyugal, por nivel de instrucción superior* y sexo

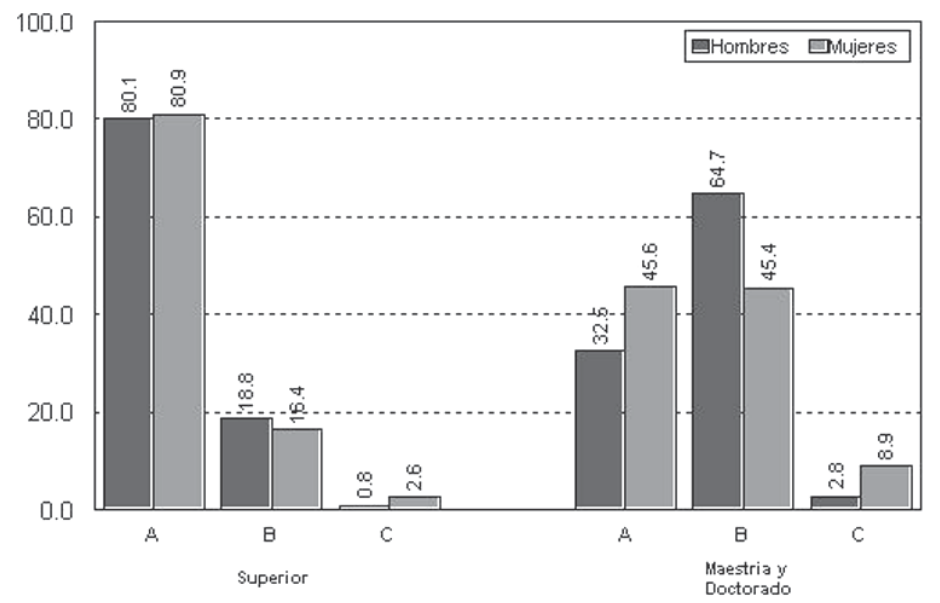

A) Soltero(a).

B) Unión libre o casado(a).

C) Separado(a) o viudo(a) o divorciado(a).

* Incluye carrera técnica o comercial con antecedentes de preparatoria terminada.

Fuente: Reprocesamiento con base en la muestra censal del XII Censo General de Población y Vivienda, 2000.

${ }^{14}$ Más adelante se considera la participación en la actividad económica de aquellos que acuden a la universidad. 


\subsection{Parentesco con el jefe de hogar}

La distribución de la población de 18 años y más, según el parentesco que guarda con el jefe del hogar en que vive, difiere entre mujeres y hombres debido a que se trata de edades cuando el hombre es mayoritaria y tradicionalmente el jefe del hogar y la mujer es cónyuge. No obstante, cuando se trata de población con estudios superiores, observamos un comportamiento menos tradicional. Entre aquellos con asistencia escolar en el 2000 en el nivel profesional, alrededor de 60\% son hijos (56.4\% y 61.2\% para hombres y mujeres, respectivamente). Esa proporción es menor cuando el grado es maestría o doctorado, pues alcanza hasta $16.3 \%$ entre los hombres y $30.3 \%$ entre las mujeres (véase la gráfica IX), lo que sugiere una situación más favorable para los estudios de las mujeres, sobre todo en los casos en que no estén participando en la actividad económica.

Llama la atención que, entre quienes ya no asisten a las instituciones de educación superior, la proporción de hijos, aunque menor, es todavía importante: $23 \%$ entre los hombres y $32.2 \%$ entre

\section{Gráfica IX}

Distribución porcentual de la población de 18 años y más con aisstencia escolar en el 2000 según parentesco con el jefe del hogar por nivel de instrucción superior* y sexo

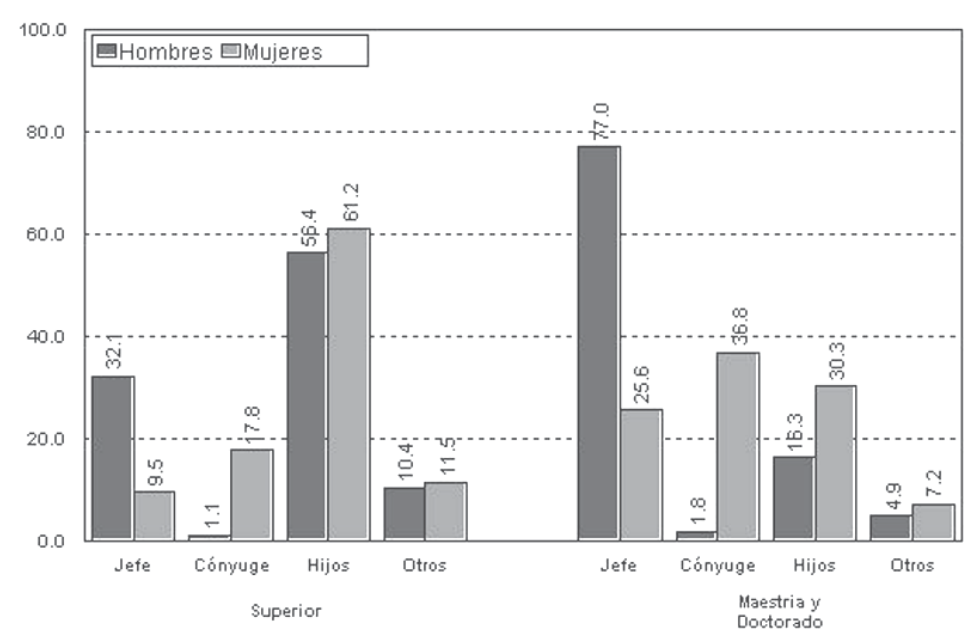

* Incluye carrera técnica o comercial con antecedentes de preparatoria terminada. Fuente: Reprocesamiento con base en la muestra censal del XII Censo General de Población y Vivienda, 2000. 
las mujeres cuando se trata del nivel profesional, y 9.3 y 20.5, respectivamente, para hombres y mujeres en el nivel de maestría o doctorado. Esto puede sugerir que la población con estudios superiores retrasa la salida del hogar paterno debido a un retraso también en la entrada a la primera unión, que es la causa tradicional para la salida del hogar junto con la migración laboral. En efecto, asistir a la universidad proporciona nuevos elementos para evaluar la opción de unirse o ser padre o madre, lo que puede resultar en la postergación de esos eventos por un largo plazo. Para los individuos, esto se traduce en un mayor tiempo de formación y, en consecuencia, mayores oportunidades laborales.

La jefatura del hogar atañe sólo a $32 \%$ de los hombres que asisten a la enseñanza profesional y a $9.5 \%$ de las mujeres; para ellas es más importante la proporción de cónyuges (18.8\%). Es posible que estas proporciones correspondan a la población más envejecida que asiste a la universidad, es decir, a aquellos de entrada tardía o permanencia larga, justamente por dedicarse al menos a dos actividades a la vez (familia y estudios).

En el nivel de maestría o doctorado, la mayoría de los hombres son jefes de hogar, ya sea con o sin asistencia escolar a las instituciones de educación superior (77 y 85\%, respectivamente), lo cual corresponde al rol tradicional masculino para esas edades adultas. Entre las mujeres, la proporción de jefas es importante y alcanza $26 \%$ en condiciones de asistencia y $22 \%$ cuando ya no se asiste a la enseñanza superior. Es interesante notar que ambas proporciones son mayores a la de jefas de hogar respecto del total de hogares en el país: $20.6 \%$ de hogares (INEGIInmujeres, 2000), lo que sugiere que precisamente la formación profesional permite a las mujeres afrontar la responsabilidad de encabezar su hogar.

\subsection{Escolaridad del jefe del hogar}

La valoración de la educación en el entorno familiar es una condición que favorece el ingreso y permanencia en las instituciones educativas en los distintos niveles, ya que supone un ambiente en el que se tiene, por ejemplo, acceso a bienes culturales, como libros y revistas que promueven la adquisición de nuevos conocimientos e ideas.

La escolaridad de los padres es un buen indicador del valor que se da a los altos niveles de formación. Como puede observarse en la gráfica $\mathrm{x}$, la proporción de jefes de hogar con estudios superio- 


\section{Gráfica $\mathrm{X}$}

Distribución porcentual del total de hogares y de aquellos con hijos que asisten a instituciones de educación superior según nivel de instrucción del jefe, 2000

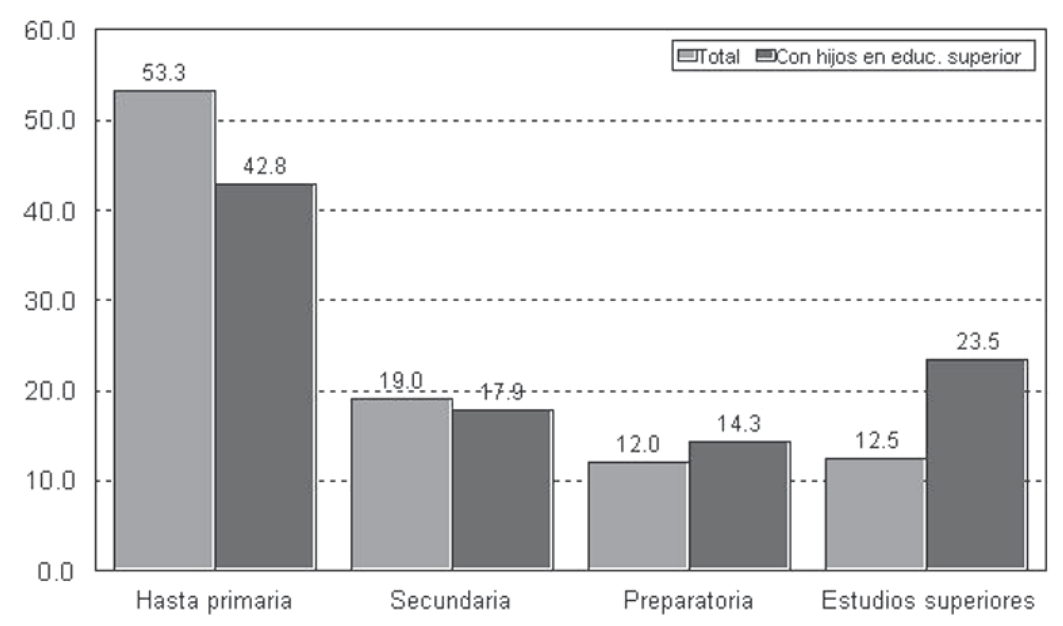

Fuente: Reprocesamiento con base en la muestra censal del XII Censo General de Población y Vivienda, 2000.

res y padres de hijos(as) que asistían a alguna institución de educación superior en el 2000 duplica a la proporción de jefes con educación superior del total de hogares del país. Esa situación da cuenta de que un ambiente donde se vive cotidianamente con los beneficios de tener estudios profesionales es más propenso para que los hijos alcancen el nivel de educación superior.

\section{Estudiar solamente es un lujo}

Dedicarse a estudiar tiempo completo parece ser un lujo de pocos. La proporción de la población que se dedica solamente a los estudios de licenciatura o profesional sin tener que trabajar es de $45 \%$ entre los hombres y de $57 \%$ entre las mujeres. Cuando se alcanza el nivel de maestría o doctorado, esta proporción se reduce a $12.5 \%$ entre los hombres y $23.1 \%$ entre las mujeres (véase la gráfica XI).

Es importante señalar que las facilidades para ingresar a la universidad y la flexibilidad del funcionamiento institucional permiten a los individuos combinar el trabajo o el matrimonio con los estudios. Esto se observa en los programas universitarios que ofrecen opciones para las personas que trabajan o que, por algu- 


\section{Gráfica XI}

Porcentaje de población económicamente inactiva respecto de la población con al menos un año de educación superior, 2000

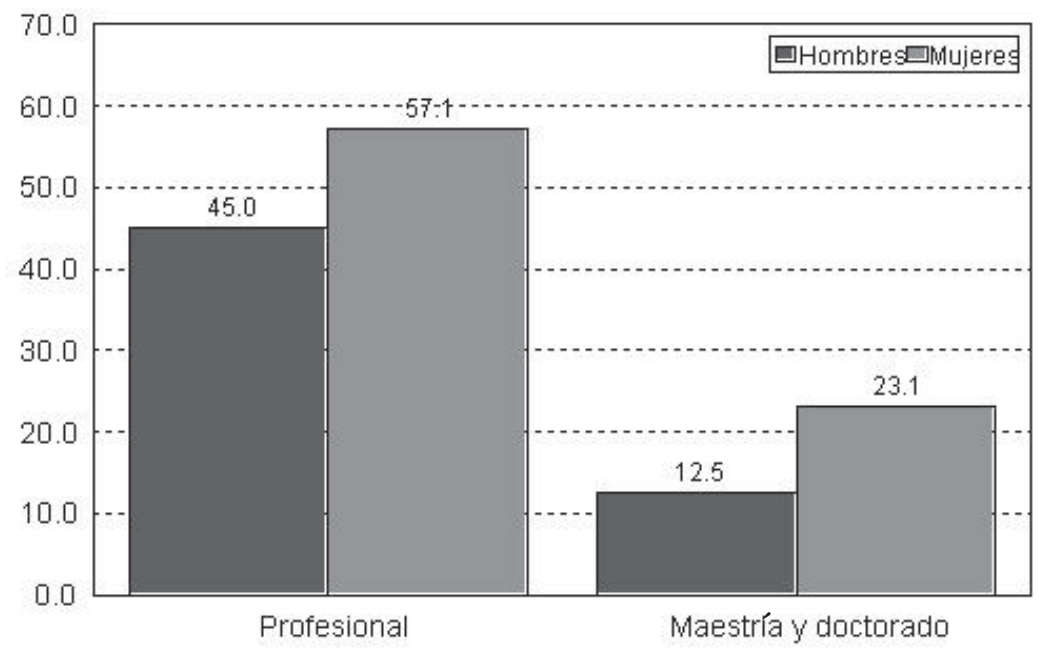

* Incluye carrera técnica o comercial con antecedentes de preparatoria terminada. Fuente: Reprocesamiento con base en la muestra censal del XII Censo General de Población y Vivienda, 2000.

na razón, no pueden dedicarse exclusivamente a los estudios. Ejemplo de ello son las licenciaturas abiertas. Sin embargo, la realidad ha mostrado que combinar distintas opciones no es fácil, particularmente en situaciones de crisis económicas, puesto que cambian los costos de oportunidad y las opciones tienden a ser excluyentes.

Al parecer, las mujeres se ven mayormente favorecidas para dedicarse de tiempo completo a los estudios. Sin embargo, al considerar otros factores como el estado conyugal, el parentesco con el jefe de hogar y el hecho de tener o no tener hijos, es posible que, en muchos casos, la dedicación de las mujeres a los estudios se combine con sus roles de género tradicionales de madre y cónyuge, o bien, de jefa de hogar, particularmente cuando se estudia fuera de una trayectoria regular.

De acuerdo con los datos de la muestra censal del 2000, entre las mujeres de 18 a 24 años con asistencia escolar a alguna institución de educación superior, apenas alrededor del 6\% tiene hijos. Cuando las mujeres tienen 25 años o más, la proporción de madres entre las estudiantes universitarias alcanza 50\%. Tomando en cuenta que $40 \%$ de las mujeres que asisten a la universidad tiene 25 años 
o más, y que la mitad de ellas tiene hijos, entonces tenemos que una quinta parte de las asistentes a la universidad son madres.

\section{Participación económica}

Contar con educación superior supone no sólo un instrumento para participar activamente en la economía, sino también para acceder a posiciones de alto nivel en el mercado laboral. Esto último puede no ser cierto, pero sí lo primero. La población que ha accedido a la educación superior muestra una mayor tasa de participación económica comparada con la de la población total de 18 años y más. Esto es particularmente evidente entre las mujeres con educación profesional cuya tasa de participación económica casi duplica la tasa promedio femenina cuando ya no se asiste a las instituciones de educación superior (véase la gráfica XII).

En el nivel de maestría o doctorado, ya sea en condiciones de asistencia o no asistencia escolar, esa tasa se incrementa sensi-

\section{Gráfica XII}

Tasas de participación en la actividad económica de la población de 18 años y más por el nivel de instrucción superior*, condición de asistencia escolar y sexo, 2000

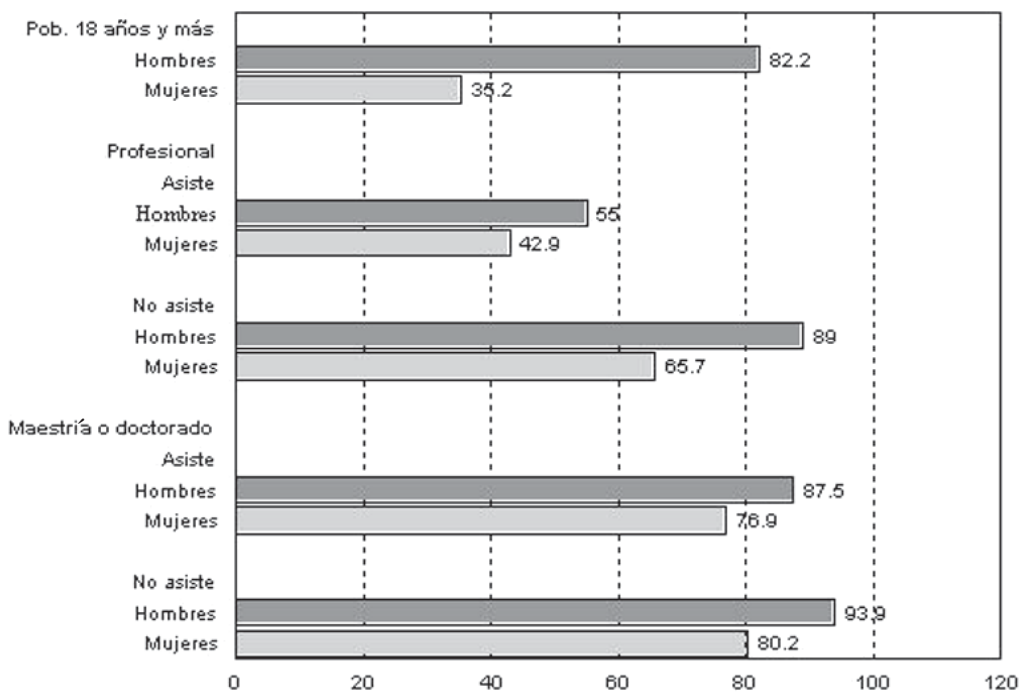

* Incluye carrera técnica o comercial con antecedentes de preparatoria terminada. Fuente: Reprocesamiento con base en la muestra censal del XII Censo General de Población y Vivienda, 2000. 
blemente y se acerca a la tasa de participación económica del total de hombres de 18 años y más.

\section{Conclusiones}

A lo largo de este análisis pudimos observar cómo mujeres y hombres aprovecharon un escenario de creciente valoración de la educación, particularmente la de nivel superior, y en el que las oportunidades para obtener ese tipo de formación se han extendido y han tocado a sectores de toda la escala social. Particularmente, encontramos una creciente participación de las mujeres en la formación profesional superior, la cual, incluso, llega a rebasar la participación masculina en las generaciones más jóvenes.

Este aumento no ocurrió solamente en aquellas áreas de estudio en las cuales las mujeres habían tenido importante participación. Los cambios en la relación de feminidad evidencian cómo dicho aumento permeó todas las áreas, lo que nos permite pensar que, quizá en un futuro no muy lejano, dejaremos de hablar de profesiones 'feminizadas'.

La participación en la educación superior se ha incrementado a pesar de que las crisis económicas han afectado las condiciones de bienestar de las familias y que éstas se han visto obligadas a realizar esfuerzos importantes y costosos para que sus hijos se formen en el nivel superior. Consideramos que esto se debe a que los padres de los que han accedido a la instrucción superior también habían escalado niveles educativos, lo cual les implicó una mayor valoración de la instrucción superior. En este sentido, el análisis mostró que el ingreso a los estudios superiores es más frecuente entre los hijos(as) de jefes de hogar con ese mismo nivel de estudios.

También se encontró que la participación en el mercado de trabajo es común entre la población con asistencia escolar a alguna institución de educación superior, lo cual puede estar afectando el desempeño en los estudios dado que la responsabilidad de trabajar para aportar ingresos al hogar compite, con ventaja, en los esfuerzos que implica la formación profesional.

La participación en la actividad económica es cada vez más frecuente como antecedente al ingreso a la universidad; esto parece evidenciarse con la importante proporción de población que asiste a la universidad, en nivel profesional, después de los 24 años, lo que refleja la postergación de los estudios superiores.

El desarrollo de los estudios superiores a la par de la actividad económica tiene, evidentemente, implicaciones serias para 
el desempeño de los propios estudios, ya que puede alargar el tiempo para realizarlos, o bien, provocar una deserción en el largo plazo. En este sentido, encontramos el menor riesgo entre las mujeres que asisten a las instituciones de educación superior dado que presentan una menor participación en la actividad económica respecto de los hombres. Pensamos, al igual que Muñiz (1997), que precisamente por las diferencias de género, las mujeres pueden haberse visto favorecidas para continuar sus estudios superiores, ya que si la familia requiere mayores ingresos, los primeros obligados a abandonar la escuela son los varones, a quienes se les exigiría cumplir con su rol de proveedores.

Haber alcanzado niveles de educación superior ha generado entre las mujeres una mayor participación económica, sobre todo una vez que se ha dejado de asistir a las instituciones de educación superior. Estas dos variables, en conjunto, permiten a las mujeres mayor autonomía y las pone en una mejor posición para negociar tanto en el ámbito familiar como en el laboral. Por ello, es de celebrarse que en la educación superior la matrícula de mujeres y hombres haya avanzado hacia la paridad.

\section{Bibliografía}

ANUIES (Asociación Nacional de Universidades e Instituciones de Educación Superior) (2002), Anuario estadístico 2001. Población escolar de licenciatura en universidades e institutos tecnológicos, ANUIEs, México.

Conmujer (Comisión Nacional de la Mujer) et al. (2001), El enfoque de género en la producción de las estadísticas educativas de México. Conmujer, FNUAP, OPS/OMS, PNUD, UNICEF e INEGI, México.

Cortés, F. y R. M. Ruvalcaba (1991), Autoexploración forzada y equidad por empobrecimiento: la distribución del ingreso familiar en México (1977-1984), El Colegio de México, México.

Díaz Cossío, R., L. M. Matamoros y A. Cerón Roa (2000), "Eficiencia de las Instituciones Mexicanas de Educación Superior", http://www.sesic.sep.gob.mx/eimes2000/ index.htm. 
INEGI (Instituto Nacional de Estadística, Geografía e Informática) (2000), XII Censo General de Población y Vivienda, 2000. Muestra censal, cuestionario ampliado, México.

e Inmujeres (Instituto Nacional de las Mujeres) (2000), Mujeres y hombres, México.

Muñiz Martelón, P. E. (1997), Trayectorias educativas y deserción universitaria en los ochenta, ANUIES, colección Temas de Hoy en la Educación Superior, México.

Pérez Franco, L. (1998). "Los factores socioeconómicos que inciden en el rezago y la deserción escolar", http://www. anuies.mx/anuies/libros98/lib64/5.html.

PNUD (Programa de las Naciones Unidas para el Desarrollo) (2001), Informe Mundial de Desarrollo Humano 2001, PNUD, http:/ /www.undp.org./hdr2001/spanish/spidhtod.pdf, 17 de noviembre de 2004.

SEP (Secretaría de Educación Pública) (2001), Programa Nacional de Educación 2001-2006, sEP, México.

Tuirán, R. et al. (2002). “Tendencias y perspectivas de la fecundidad", La situación demográfica de México, 2002, Consejo Nacional de Población (Conapo), México, pp. 29-48.

Enviado: 29 de marzo de 2004. Aceptado: 17 de noviembre de 2004.

Silvia Luna Santos es candidata a doctora en demografía por la Université Paris X-Nanterre, maestra en demografía por El Colegio de México. Sus líneas de investigación actuales se orientan a la familia, trayectorias familiares, relaciones de parentesco y situación socioeconómica de las mujeres-madres. 